\title{
Risk of Rebleeding in Patients with Small Bowel Vascular Lesions
}

\author{
Akira Harada $^{1}$, Takehiro Torisu ${ }^{1}$, Shin Fujioka ${ }^{1}$, Yuichiro Yoshida ${ }^{1}$, Yasuharu Okamoto ${ }^{1}$, \\ Yuta Fuyuno ${ }^{1}$, Atsushi Hirano ${ }^{1}$, Junji Umeno ${ }^{1}$, Kumiko Torisu ${ }^{1}$, Tomohiko Moriyama ${ }^{1}$, \\ Motohiro Esaki ${ }^{2}$ and Takanari Kitazono ${ }^{1}$
}

\begin{abstract}
:
Background With recent advances in endoscopic modalities, small bowel vascular lesions (SBVLs) are often now detected in patients with gastrointestinal bleeding. Given the high invasiveness of endoscopic treatment, it is important to select patients at high risk for bleeding.

Aim To assess the risk of rebleeding in patients with SBVLs as a systemic disease rather than a gastrointestinal disease in relation to their general health.

Methods We retrospectively analyzed the clinical data of 55 patients with SBVLs among patients with obscure gastrointestinal bleeding. The possible association between the clinical findings and the updated Charlson comorbidity index with rebleeding was evaluated.

Results Gastrointestinal rebleeding occurred in 20 patients (36.4\%) during the follow-up period. The presence of multiple comorbidities as indicated by an updated Charlson comorbidity index of $\geq 4$ was a risk factor for rebleeding (hazard ratio, 3.64; $\mathrm{P}=0.004$ ). Other risk factors were arteriosclerosis of the superior mesenteric artery and multiple SBVLs. Endoscopic hemostasis and the discontinuation of antithrombotic medications were not significantly associated with rebleeding. Patients with a high updated Charlson comorbidity index had a high risk of death of causes other than gastrointestinal rebleeding.

Conclusions Gastrointestinal rebleeding is not a rare condition among patients with SBVLs. Patients with poor general health may therefore have a higher risk of rebleeding.
\end{abstract}

Key words: angioectasia, arteriosclerosis, capsule endoscopy, Charlson comorbidity index, rebleeding, small bowel

(Intern Med Advance Publication)

(DOI: 10.2169/internalmedicine.6341-20)

\section{Introduction}

Small bowel vascular lesions (SBVLs) are most frequently identified in patients with obscure gastrointestinal bleeding (OGIB) (1). The advent of capsule endoscopy (CE) and balloon-assisted enteroscopy (BAE) has enabled the detailed observation of the small intestine and has increased the opportunities to detect SBVLs (2). CE is a suitable modality for screening the entire small intestine in a minimally invasive manner (3). BAE is useful for both endoscopic treatment and the diagnosis of SBVLs $(2,4)$. Despite this progress in the development of devices for small intestinal disease, recurrent bleeding from SBVLs is a clinical problem. In most patients with SBVLs, bleeding recurs even after hemostasis has been obtained (5). Although the preventive effect of BAE against rebleeding is unclear, BAE is too invasive for elderly patients and patients with comorbidities (2). When determining the indications for BAE, it is necessary to consider the balance between general health and the risk of rebleeding $(4,6,7)$.

In order to evaluate patients' general health, the updated

${ }^{1}$ Department of Medicine and Clinical Science, Graduate School of Medical Sciences, Kyushu University, Japan and ${ }^{2}$ Divison of Gastroenterology, Department of Internal Medicine, Faculty of Medicine, Saga University Hospital, Japan

Received: September 18, 2020; Accepted: April 22, 2021; Advance Publication by J-STAGE: June 12, 2021

Correspondence to Dr. Takehiro Torisu, torisut@intmed2.med.kyushu-u.ac.jp 


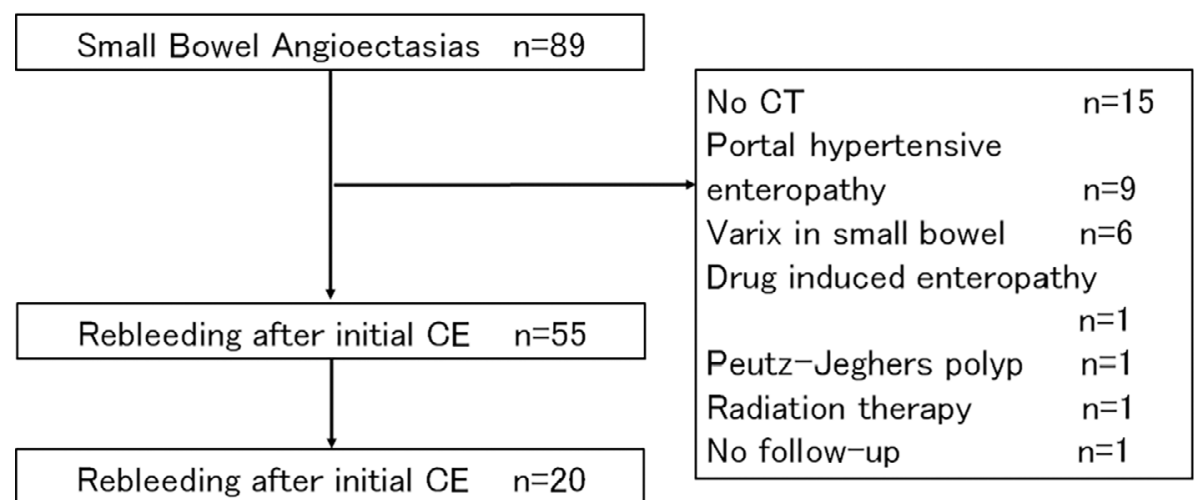

From January 2004 to December 2018 in Kyushu University Hospital

Figure 1. A study flow diagram showing the clinical course of the overall patients and the exclusion criteria.

Charlson comorbidity index (uCCI) is widely used $(8,9)$. The CCI was designed to predict post-discharge mortality, and the uCCI has been updated for the current medical care situation (10). Arteriosclerosis and sarcopenia are associated with multiple comorbidities and general frailty (11). In addition, these factors are related to the morbidity of SBVLs (12). Therefore, we evaluated the relation between rebleeding and general health in patients with SBVLs.

The present study was performed to identify the risk factors for rebleeding in patients with OGIB diagnosed with SBVLs among various underlying diseases. We evaluated the uCCI and sarcopenia to reveal the association between bleeding and the general health condition.

\section{Materials and Methods}

\section{Patients}

Fig. 1 shows a study flow diagram. All patients who had given their informed consent and been underwent $\mathrm{CE}$ were registered in CE data base in Kyushu University Hospital. From January 2004 to December 2018, 89 patients with OGIB underwent $\mathrm{CE}$ after conventional upper and lower endoscopy and were diagnosed with SBVLs. In our study SBVLs were defined as type $1 \mathrm{a}, 1 \mathrm{~b}, 2 \mathrm{a}$ and $2 \mathrm{~b}$ in YanoYamamoto classification (13), since diminutive SBVLs, even classified as type 1a, could cause massive bleeding intermittently $(4,14)$.

Since the purpose of this study was to assess the balance between the risk of rebleeding and the general health in order to consider the indications for BAE, patients with diseases for which other treatments were preferred over hemostasis with BAE or with insufficient information were excluded. To be more specific, those who met the following criteria were excluded from the study: patients who did not undergo a CT scan within 1 month from $\mathrm{CE}$, and patients without any follow-up information, patients with varix in small bowel, drug-induced enteropathy, Peutz-Jeghers polyp, a history of radiation therapy and portal hypertensive entero- pathy (15). Finally, the remaining patients with SBVLs were included in this study.

The ethics committee of Kyushu University Hospital approved the research protocol (UMIN Test ID: UMIN 000030027), and the study was conducted according to the Declaration of Helsinki. Prior to testing, written informed consent was obtained from all participants.

\section{Data collection}

The following clinical information was retrospectively collected from the patients' charts: clinical features, underlying disease, CT findings, CE findings, SBVL findings, oral medications (low-dose aspirin [LDA], anticoagulants, antiplatelets [excluding LDA], and nonsteroidal antiinflammatory drugs [excluding LDA]), lowest hemoglobin concentration before $\mathrm{CE}$, interval between first $\mathrm{CE}$ examination and rebleeding, and discontinuation of antithrombotic drugs. A hemoglobin concentration of $<7.0 \mathrm{~g} / \mathrm{dL}$ was defined as severe anemia, overt OGIB was defined as melena or bloody stool, and occult OGIB was defined as a positive stool occult blood test with iron deficiency anemia or anemia. The uCCI was calculated from the underlying diseases according to a previous report (8).

\section{CE examination}

The PillCam SB 2 or SB 3 (Medtronic, Dublin, Ireland) and M2A or Pillcam SB(Given Imaging, Yokneam, Ireland) were used to perform $\mathrm{CE}$ according to the manufacturer's recommendations. After fasting for 12 hours overnight, each patient underwent preparation with magnesium citrate or simethicone. Each patient was instructed to position the sensor array and data recorder and swallow the capsule with a small amount of water. CE images were usually recorded in a real-time viewer for more than 8 hours. All digital video image streams were downloaded to an image and data reporting and processing system (Medtronic) and evaluated by three experienced CE observers (A.H., Y.O., and M.E.). The video images were analyzed at an average rate of 15 to 20 frames/second and then were subjected to simultaneous 


\section{A; Aorta \\ B; celiac artery \\ C; superior mesenteric artery}
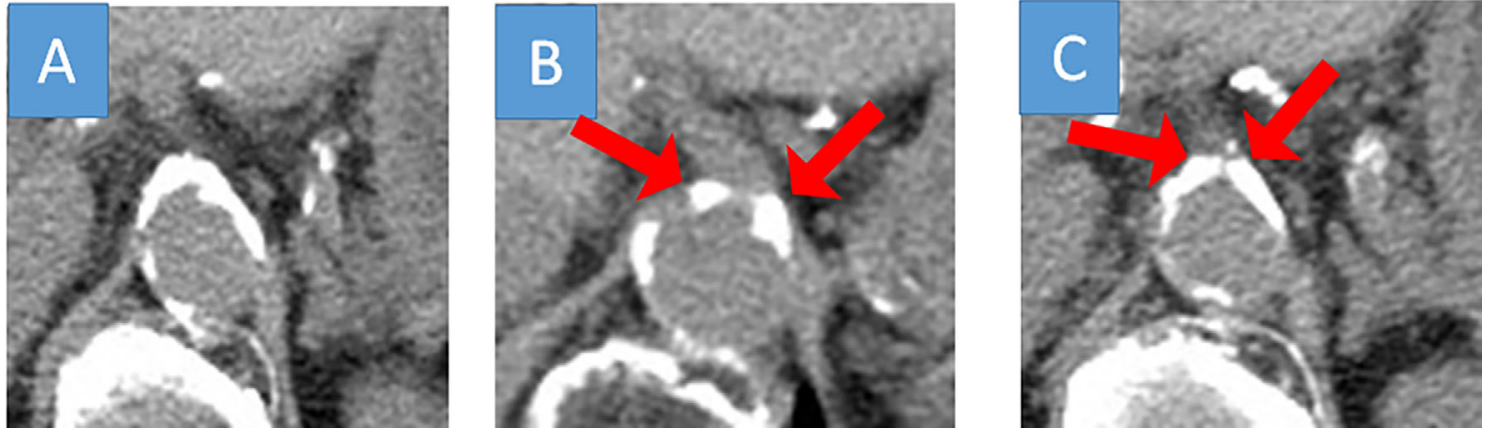

Figure 2. Arteriosclerosis of the main abdominal arteries. (A) Aorta. (B) Celiac artery (arrow). (C) Superior mesenteric artery (arrow).

manual inspection for precise evaluation on a multiview system (6).

\section{Evaluation of the abdominal CT findings}

Abdominal CT was performed with a 32- to 64-row multidetector CT scanner (Aquilion; Canon Medical Systems, Tochigi, Japan). Of 55 patients, a simple radiograph was obtained in 38 and contrast enhancement was used in 17. Calcification of the abdominal aorta, celiac artery, and superior mesenteric artery was confirmed based on a horizontal image taken at a width of $5 \mathrm{~mm}$. Arteriosclerosis of the abdominal aorta was defined as clear calcification of more than half the circumference of the lumen. According to a previous report (16), arteriosclerosis of the celiac artery and superior mesenteric artery was defined as clear calcification on both sides of the blood vessel at origin of artery (Fig. 2). Sarcopenia can be evaluated by the iliopsoas area using a minimally invasive CT scan (17). The iliopsoas area at the L 3 level was measured using the manual trace method, and the iliopsoas area/height squared was calculated as the psoas muscle mass index. Sarcopenia was defined as a psoas muscle mass index of $\leq 6.36 \mathrm{~cm}^{2} / \mathrm{m}^{2}$ in men and $\leq 3.92 \mathrm{~cm}^{2} / \mathrm{m}^{2}$ in women (17).

\section{Study endpoints}

The endpoint of this study was rebleeding after the initial CE. Rebleeding was defined as melena, hematochezia, positive fecal occult blood or iron deficiency anemia with a decrease in $\mathrm{Hb}$ levels 2 or more $\mathrm{g} / \mathrm{dL}$.

\section{Statistical analysis}

All analyzes were performed using the JMP version 14.0.2 software program (SAS Institute Inc., Tokyo, Japan). Categorical variables are presented as the frequency and percentage, and numerical variables are presented as the mean or median. The cumulative rates of rebleeding and death after CE were calculated by the Kaplan-Meier method. Factors associated with rebleeding and mortality were evaluated using a Cox proportional hazards model. A $\mathrm{P}$ value of $<0.05$ was considered to be statistically significant.

\section{Results}

\section{Characteristics of the patients at initial CE}

Table 1 summarizes the clinical characteristics of the 55 patients at the initial CE examination. The patients comprised 29 men and 26 women with ages ranging from 32 to 91 years (median, 72 years). The hemoglobin level at the time of CE ranged from 4.1 to $11.7 \mathrm{~g} / \mathrm{dL}$ (median, $7.5 \mathrm{~g} /$ $\mathrm{dL}$ ), which was defined as severe anemia in 22 patients. The uCCI calculated from these comorbidities ranged from 0 to 8 (median, 2).

CT showed calcification in the aorta in 24 patients, the celiac artery in 26, and the superior mesenteric artery in 24. With respect to muscle mass, the psoas muscle mass index ranged from 2.23 to $7.82 \mathrm{~cm}^{2} / \mathrm{m}^{2}$ (median, $4.61 \mathrm{~cm}^{2} / \mathrm{m}^{2}$ ), and 33 patients met the sarcopenia criteria.

During the CE examinations, SBVLs were detected in 1 to 7 lesions (median, 2). A total of 42 patients showed lesions in the jejunum and 28 in the ileum. Treatment by BAE was performed in 13 patients by argon plasma coagulation, in six patients by clipping and in seven patients by both devices.

\section{Factors associated with rebleeding during follow-up}

After the initial CE twenty of 55 patients rebled. The cumulative rebleeding rates are shown in Figure 3 (median duration, 22 months; range, 1-96 months). The cumulative rebleeding rates after 12,24 , and 45 months were $32.6 \%$, 
Table 1. Clinical Characteristics of Patients with Vascular Lesions in the Small Bowel.

\begin{tabular}{|c|c|}
\hline & $\mathrm{n}=55$ \\
\hline Age, years, median (range) & $72(32-91)$ \\
\hline Male sex, n (\%) & $29(52.7)$ \\
\hline $\mathrm{BMI} \geq 25, \mathrm{~kg} / \mathrm{m}^{2}$, median (range) & $21.1(13.8-36.7)$ \\
\hline Smoking current/past/never, $\mathrm{n}$ & $12 / 16 / 27$ \\
\hline Drinking current/past/never, $\mathrm{n}$ & $16 / 13 / 26$ \\
\hline \multicolumn{2}{|l|}{ Comorbidities } \\
\hline Hypertension, n (\%) & $31(56.4)$ \\
\hline Ischemic heart diseases, $\mathrm{n}(\%)$ & $17(30.9)$ \\
\hline Heart failure, n $(\%)$ & $10(18.1)$ \\
\hline Cerebrovascular diseases, $\mathrm{n}(\%)$ & $9(16.4)$ \\
\hline Diabetes Mellitus, n (\%) & $15(27.3)$ \\
\hline Diabetes Mellitus with chronic complications, $\mathrm{n}(\%)$ & $5(9.1)$ \\
\hline Hyperlipidemia, n (\%) & $19(34.5)$ \\
\hline Hemodialysis, $\mathrm{n}(\%)$ & $10(18.1)$ \\
\hline Chronic kidney disease, $\mathrm{n}(\%)$ & $26(47.3)$ \\
\hline Liver cirrhosis, n (\%) & $7(12.7)$ \\
\hline Malignant tumors, n (\%) & $7(12.7)$ \\
\hline Updated CCI $\geq 4$, median (range) & $2(0-8)$ \\
\hline \multicolumn{2}{|l|}{ Drugs } \\
\hline LDA, n $(\%)$ & $23(41.8)$ \\
\hline Aps, n (\%) & $13(23.6)$ \\
\hline Acs, n (\%) & $15(27.3)$ \\
\hline NSAIDs, n (\%) & $2(3.6)$ \\
\hline Discontinued antithrombotic, $\mathrm{n}(\%)$ & $6(10.9)$ \\
\hline $\mathrm{Hb}, \mathrm{g} / \mathrm{dL}$, median (range) & $4.1(7.5-11.7)$ \\
\hline Overt-OGIB, n (\%) & $41(74.5)$ \\
\hline \multicolumn{2}{|l|}{ Arteriosclerosis } \\
\hline Aorta, n (\%) & $24(43.6)$ \\
\hline Celiac artery, n (\%) & $24(43.6)$ \\
\hline Superior mesenteric artery, $\mathrm{n}(\%)$ & $26(47.2)$ \\
\hline Psoas muscle index $\left(\mathrm{cm}^{2} / \mathrm{m}^{2}\right)$ & $2.23-7.82(4.41)$ \\
\hline \multicolumn{2}{|l|}{ SBVLs findings under CE } \\
\hline Number of SBLs & $2(1-7)$ \\
\hline jejunum, n (\%) & $42(76.4)$ \\
\hline ileum, n (\%) & $28(50.9)$ \\
\hline Endoscopic treatment, $\mathrm{n}(\%)$ & $26(47.2)$ \\
\hline
\end{tabular}

BMI: body mass index, CCI: Charlson comorbidity index, LDA: low-dose aspirin, Aps: non-aspirin antiplatelets, Acs: anticoagulants, NSAIDs: nonsteroidal anti-inflammatory drugs, Hb: hemoglobin, OGIB: obscure gastrointestinal bleeding, SBVLs: small bowel vascular lesions, CE: capsule endoscopy

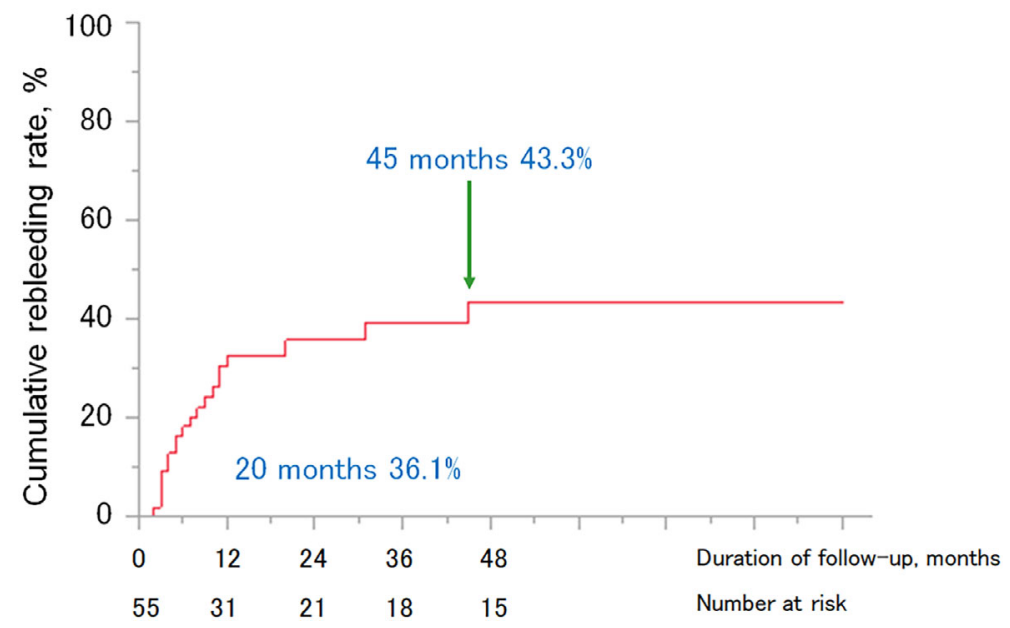

Figure 3. Kaplan-Meier curves showing the cumulative rebleeding rates. 
Table 2. A Univariate Analysis of the Risk Factors for Rebleeding in Patients with Small Bowel Angioectasias.

\begin{tabular}{|c|c|c|c|}
\hline & Hazard ratio & $95 \% \mathrm{Cl}$ & $\mathrm{p}$ value \\
\hline Age $\geq 70$ years & 0.66 & $0.28-1.60$ & 0.36 \\
\hline Male sex & 1.08 & $0.45-2.61$ & 0.87 \\
\hline $\mathrm{BMI} \geq 25$ & 0.32 & $0.04-2.41$ & 0.32 \\
\hline Smoking (current/past) & 1.79 & $0.70-4.53$ & 0.22 \\
\hline Drinking (current/past) & 1.32 & $0.51-2.93$ & 0.44 \\
\hline Updated CCI $\geq 4$ & 3.64 & $1.50-8.84$ & 0.004 \\
\hline \multicolumn{4}{|l|}{ Comorbidities } \\
\hline Hypertension & 0.83 & $0.33-2.03$ & 0.68 \\
\hline Ischemic heart diseases & 1.31 & $0.53-3.31$ & 0.56 \\
\hline Heart failure & 2.17 & $0.83-5.66$ & 0.11 \\
\hline Cerebrovascular diseases & 0.96 & $0.83-5.66$ & 0.95 \\
\hline Diabetes mellitus & 0.64 & $0.28-3.29$ & 0.43 \\
\hline Hyperlipidemia & 0.65 & $0.21-1.92$ & 0.40 \\
\hline Chronic kidney disease & 1.38 & $0.54-3.50$ & 0.50 \\
\hline Liver cirrhosis & 2.01 & $0.67-6.04$ & 0.22 \\
\hline Malignant tumors & 1.35 & $0.38-4.78$ & 0.64 \\
\hline \multicolumn{4}{|l|}{ Drugs } \\
\hline LDA & 0.70 & $0.28-1.75$ & 0.36 \\
\hline Aps & 0.66 & $0.22-1.97$ & 0.45 \\
\hline Acs & 1.59 & $0.63-3.99$ & 0.33 \\
\hline Discontinued anti-thrombotics & 0.86 & $0.20-3.73$ & 0.84 \\
\hline Severe anemia & 1.77 & $0.73-4.28$ & 0.20 \\
\hline Overt-OGIB & 0.96 & $0.35-2.65$ & 0.96 \\
\hline \multicolumn{4}{|l|}{ Arteriosclerosis } \\
\hline Aorta & 1.15 & $0.48-2.79$ & 0.75 \\
\hline Celiac artery & 1.40 & $0.53-3.53$ & 0.47 \\
\hline Superior mesenteric artery & 2.56 & $1.02-6.43$ & 0.05 \\
\hline Sarcopenia & 0.92 & $0.37-2.27$ & 0.87 \\
\hline \multicolumn{4}{|l|}{ SBVLs findings under initial CE } \\
\hline Jejunum & 0.98 & $0.36-2.81$ & 0.98 \\
\hline Ileum & 1.84 & $0.74-4.64$ & 0.19 \\
\hline SBVLs $\geq 3$ & 2.92 & $1.21-7.07$ & 0.02 \\
\hline Endoscopic treatment & 0.84 & $0.35-2.02$ & 0.69 \\
\hline
\end{tabular}

CI: confidence interval, BMI: body mass index, CCI: Charlson comorbidity index, LDA: low-dose aspirin, Aps: non-aspirin antiplatelets, Acs: anticoagulants, OGIB: obscure gastrointestinal bleeding, SBVLs: small bowel vascular lesions, CE: capsule endoscopy

Values were estimated by Cox proportional hazards models.

$35.7 \%$, and $43.3 \%$, respectively. Univariate analyses showed a significant association between rebleeding and uCCI of $\geq 4$ $(\mathrm{P}=0.004)$ (Table 2). The log-rank test showed that patients with a uCCI of $\geq 4$ had a significantly higher rebleeding rate (Fig. 3). This result indicates that the general health status was associated with rebleeding from SBVLs, and we therefore attempted to identify underlying factors. Arteriosclerosis was evaluated using CT, and calcification of the superior mesenteric artery was associated with a significantly higher rebleeding rate $(\mathrm{P}=0.05)$, although calcification of the aorta or celiac artery was not. With respect to sarcopenia, a lower muscle mass was not a risk factor for rebleeding. The initial CE examination showed that the presence of more than three SBVLs was associated with a significantly higher rebleeding rate $(\mathrm{P}=0.02)$ (Table 2$)$.

\section{Rebleeding source and the prognosis in patients with SBVL bleeding after initial CE}

Twenty of 55 patients rebled after the initial CE and eight patients underwent repeated examinations to identify the source of re-bleeding in small bowel by CE or DBE. The source of rebleeding was identified in four patients who had SBVLs, while it could not be identified in four patients.

The cumulative survival rate after the initial CE examination is shown in Figure 4 (median, 35 months; range, 1-96 months). Thirteen patients died during the follow-up, and the cumulative survival rates at 12, 24, and 57 months were $95.6 \%, 85.3 \%$, and $53.4 \%$, respectively. The cause of death was confirmed in all 13 patients as cardiovascular disease (n $=10)$, infectious disease $(\mathrm{n}=1)$, a malignant tumor $(\mathrm{n}=1)$, 


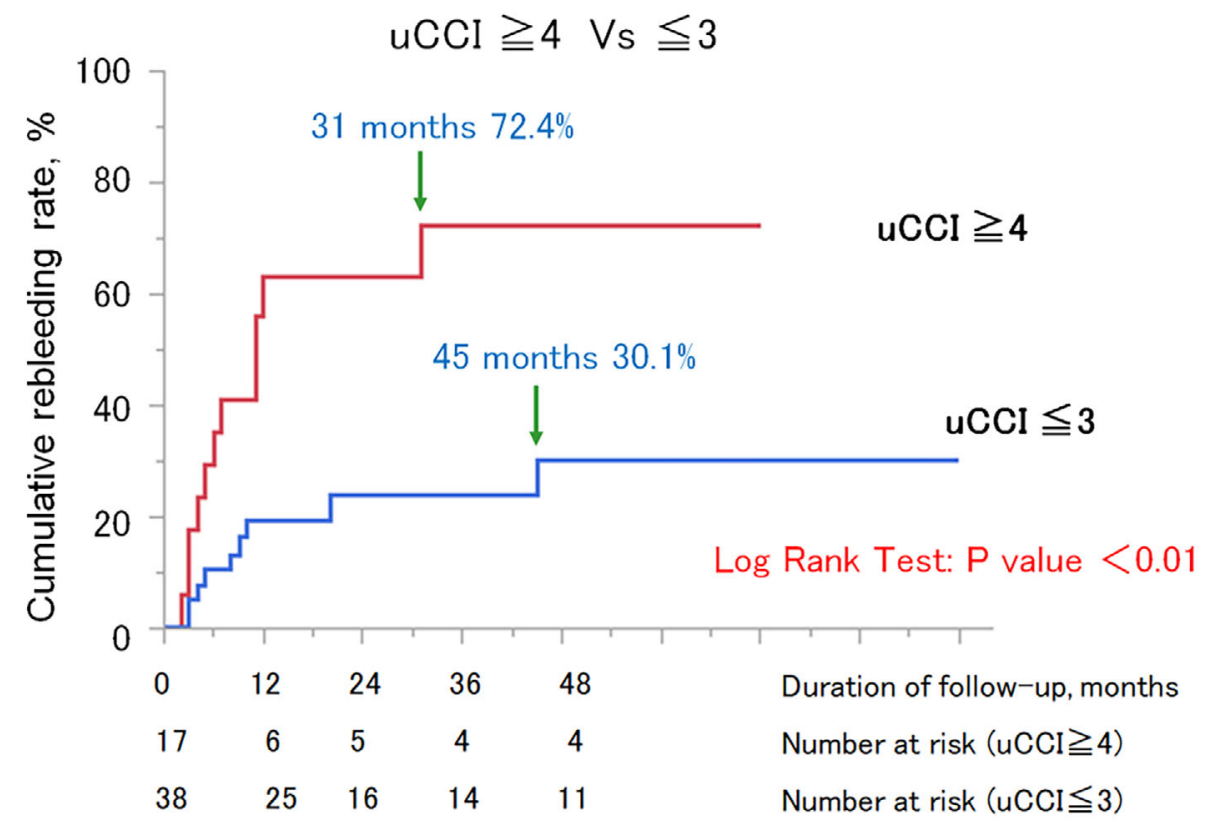

Figure 4. Kaplan-Meier curves showing the cumulative rebleeding rates for patients with a uCCI of $\geq 4$ and other groups of patients.

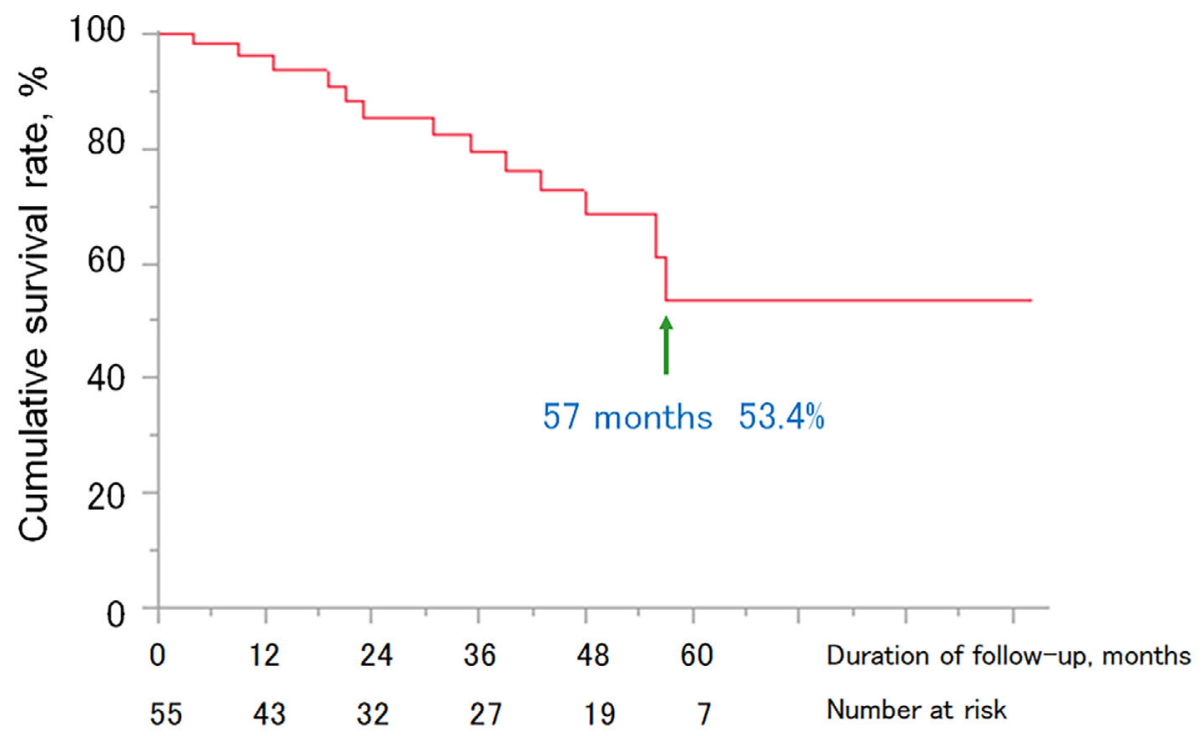

Figure 5. Kaplan-Meier curves showing the cumulative survival rates.

and gastrointestinal bleeding $(\mathrm{n}=1)$. The details of gastrointestinal bleeding were unclear because the patient was not re-examined due to a poor general condition. In the patients who died, 9 had a uCCI of $\geq 4$. Patients with a uCCI of $\geq 4$ had a significantly higher risk of death (HR, 3.60; 95\% CI, 1.10-11.78; $\mathrm{P}=0.03$ ), and patients with arteriosclerosis of the superior mesenteric artery tended to have a higher risk of death (HR, 2.84; 95\% CI, 0.86-9.35; $\mathrm{P}=0.08$ ).

\section{Discussion}

This study showed that a uCCI of $\geq 4$, which indicates poor general health, was a risk factor for rebleeding in patients with SBVLs. The uCCI is widely used to assess the general health condition caused by chronic disease (8). In total, $72.7 \%$ of the patients in our study were older than 65 years, and many had multiple comorbidities. We found no specific comorbidity involved in rebleeding. The main risk factor for SBVL rebleeding is the patient's general condition; this can be evaluated by the uCCI, which combines all diseases rather than using one specific comorbidity. The existence of SBVLs is reportedly associated with frailty and a poor prognosis (12). In patients with other types of gastrointestinal bleeding, the general condition evaluated by the CCI has been shown to be a predictor of severe diverticular bleeding (18). These reports support our finding that rebleeding from SBVLs is related to poor general health.

In this study, we showed calcification of the superior mes- 
enteric artery to be a significant risk factor for rebleeding in patients with SBVLs. The uCCI is associated with the prognosis of arteriosclerosis $(8,9)$ and sarcopenia $(11)$. Therefore, we investigated the possibility that arteriosclerosis and sarcopenia as evaluated by CT may be predictive factors for the risk of rebleeding from SBVLs. Because CT is less invasive and it is often used in patients with OGIB (2), it would be clinically meaningful to be able to predict rebleeding with CT. Other studies showed the calcification of superior mesenteric artery related to existence of vascular lesions in the small intestine $(13,19)$. Because the superior mesenteric artery supplies most parts of the small intestine, it was interesting to note that calcification of this artery rather than the aorta or celiac artery was associated with rebleeding. Ohmiya et al. suggested a new index for predicting rebleeding from small bowel vascular diseases (19). The Ohmiya index gives more weight to vascular disease than uCCI and the Ohmiya index has better sensitivity than the CCI. This finding is consistent with arteriosclerosis being a risk factor for rebleeding in our study.

Consistent with previous reports $(5,20)$, this study also showed the limited effect of endoscopic treatment to prevent rebleeding. During the follow-up period, rebleeding occurred within 2 years. UCCI was associated with death in this study. The most common cause of death was cardiovascular disease, not gastrointestinal bleeding. This suggests that patients with SBVLs should be closely followed for at least 2 years after the first bleeding event, with or without endoscopic intervention. Even if rebleeding occurs, repeated invasive therapy should be carefully considered for the patients' general condition.

Our study is associated with several limitations. First, because it was a small, single-center, retrospective study, the risk factors associated with rebleeding from SBVLs in the current study need to be validated by a large, multicenter, prospective study. Second, in order to evaluate general health we selected the patients who undergo a CT scan within 1 month from $\mathrm{CE}$, which may have led to some selection bias in the analysis of rebleeding.

In conclusion, our study showed that the main risk factor for rebleeding from SBVLs was the patients' general health condition, including arteriosclerosis. The preventive effect of endoscopic treatment against rebleeding and a poor prognosis is unclear. Rebleeding from SBVLs reflects the patient's general health condition, and invasive therapeutic interventions in patients with poor general health therefore require careful consideration.

\section{The authors state that they have no Conflict of Interest (COI).}

\section{Acknowledgement}

We thank Angela Morben, DVM, ELS, from Edanz Group (https://en-author-services.edanzgroup.com/ac), for editing a draft of this manuscript.

\section{References}

1. Raju GS, Gerson L, Das A, Lewis B. American Gastroenterological Association (AGA) Institute technical review on obscure gastrointestinal bleeding. Gastroenterology 133: 1697-1717, 2007.

2. Yamamoto $H$, Ogata $H$, Matsumoto $T$, et al. Clinical practice guideline for enteroscopy. Digestive Endoscopy 29: 519-546, 2017.

3. Lewis B, Eisen G, Friedman S. A pooled analysis to evaluate results of capsule endoscopy trials. Endoscopy 37: 960-965, 2005.

4. Igawa $\mathrm{A}$, Oka S, Tanaka S, et al. Major predictors and management of small-bowel angioectasia. BMC gastroenterology 15: 1-7, 2015.

5. Samaha E, Rahmi G, Landi B, et al. Long-term outcome of patients treated with double balloon enteroscopy for small bowel vascular lesions. American Journal of Gastroenterology 107: 240246, 2012.

6. Harada A, Torisu T, Okamoto Y, et al. Predictive Factors for Rebleeding after Negative Capsule Endoscopy among Patients with Overt Obscure Gastrointestinal Bleeding. Digestion 101: 129136, 2020.

7. Viazis N, Papaxoinis K, Vlachogiannakos J, Efthymiou A, Theodoropoulos I, Karamanolis DG. Is there a role for secondlook capsule endoscopy in patients with obscure GI bleeding after a nondiagnostic first test? Gastrointestinal endoscopy 69: 850-856, 2009.

8. Quan H, Li B, Couris CM, et al. Updating and validating the Charlson comorbidity index and score for risk adjustment in hospital discharge abstracts using data from 6 countries. American journal of epidemiology 173: 676-682, 2011.

9. Zhang F, Bharadwaj A, Mohamed MO, Ensor J, Peat G, MamasMamas A. Impact of Charlson Comorbidity Index Score on Management and Outcomes after Acute Coronary Syndrome. The American Journal of Cardiology 2020.

10. Toya Y, Endo M, Nakamura S, et al. Long-term outcomes and prognostic factors with non-curative endoscopic submucosal dissection for gastric cancer in elderly patients aged $\geq 75$ years. Gastric Cancer 22: 838-844, 2019.

11. Cruz-Jentoft AJ, Sayer AA. Sarcopenia. The Lancet 393: 26362646, 2019.

12. Robertson AR, Koulaouzidis A, Brindle WM, Robertson AJ, Plevris JN. Small bowel angioectasia as a marker of frailty and poor prognosis. Endoscopy International Open 8: E953, 2020.

13. Yano T, Yamamoto H, Sunada K, Miyata T, Iwamoto M, Hayashi $\mathrm{Y}$, et al. Endoscopic classification of vascular lesions of the small intestine. Gastrointest Endosc 67: 169-172, 2008.

14. Ohmiya N, Y1no T, Yamamoto $H$, Arakawa D, Nakamura M, Honda W, et al. Diagnosis and treatment of obscure GI bleeding at double balloon endoscopy. Gastrointest Endosc 66: 72-77, 2007.

15. Konstantinos J Dabos, Anastasios Koulaouzidis. Portal Hypertensive Enteropathy, Occult Bleeding, and Capsule Endoscopy: Where Do We Go From Here? Dig Dis Sci 59: 899-901, 2014.

16. Aoyama T, Fukumoto A, Shigita K, Asayama N, Mukai S, Nagata $\mathrm{S}$. Arteriosclerosis is a major predictor of small bowel vascular lesions. Digestive diseases and sciences 63: 723-730, 2018.

17. Hamaguchi Y, Kaido T, Okumura S, et al. Proposal for new diagnostic criteria for low skeletal muscle mass based on computed tomography imaging in Asian adults. Nutrition 32: 1200-1205, 2016.

18. Kinjo K, Matsui $T$, Hisabe $T$, et al. Risk factors for severity of colonic diverticular hemorrhage. Intestinal research 16: 458, 2018.

Yamada A, Niikura R, Kobayashi Y, et al. Risk factors for small bowel angioectasia: The impact of visceral fat accumulation. World Journal of Gastroenterology: WJG 21: 7242, 2015.

19. Ohmiya N, Nakamura M, Osaki $H$, et al. Development of a comorbidity index to identify patients with small bowel bleeding at risk for rebleeding and small bowel vascular diseases. Clin Gas- 
troenterol Hepatol 17: 896-904, 2019.

20. Sakai E, Endo H, Taguri M, et al. Frequency and risk factors for rebleeding events in patients with small bowel angioectasia. BMC gastroenterology 14: 200, 2014.
The Internal Medicine is an Open Access journal distributed under the Creative Commons Attribution-NonCommercial-NoDerivatives 4.0 International License. To view the details of this license, please visit (https://creativecommons.org/licenses/ by-nc-nd/4.0/).

(C) The Japanese Society of Internal Medicine Intern Med Advance Publication 